\title{
A tensile stage for high-stress low-strain fibre studies
}

Pauw, Brian Richard; Vigild, Martin Etchells; Mortensen, Kell; Andreasen, Jens Wenzel; Klop, Enno A.

Published in:

Journal of Applied Crystallography

Link to article, DOI:

$10.1107 /$ S0021889811042373

Publication date:

2011

Document Version

Publisher's PDF, also known as Version of record

Link back to DTU Orbit

Citation (APA):

Pauw, B. R., Vigild, M. E., Mortensen, K., Andreasen, J. W., \& Klop, E. A. (2011). A tensile stage for high-stress low-strain fibre studies. Journal of Applied Crystallography, 14(6), 1297-1299.

https://doi.org/10.1107/S0021889811042373

\section{General rights}

Copyright and moral rights for the publications made accessible in the public portal are retained by the authors and/or other copyright owners and it is a condition of accessing publications that users recognise and abide by the legal requirements associated with these rights.

- Users may download and print one copy of any publication from the public portal for the purpose of private study or research.

- You may not further distribute the material or use it for any profit-making activity or commercial gain

- You may freely distribute the URL identifying the publication in the public portal

If you believe that this document breaches copyright please contact us providing details, and we will remove access to the work immediately and investigate your claim. 
Journal of

\section{Applied Crystallography}

ISSN 0021-8898

Received 25 May 2011

Accepted 13 October 2011

\section{A tensile stage for high-stress low-strain fibre studies}

\author{
Brian R. Pauw, ${ }^{\mathrm{a}, \mathrm{b} *}$ Martin E. Vigild, ${ }^{\mathrm{a}}$ Kell Mortensen, ${ }^{\mathrm{c}}$ Jens W. Andreasen ${ }^{\mathrm{b}}$ and \\ Enno A. Klop ${ }^{d}$
}

(C) 2011 International Union of Crystallography Printed in Singapore - all rights reserved

\begin{abstract}
aDanish Polymer Centre, Department of Chemical Engineering, Technical University of Denmark, DK-2800 Kongens Lyngby, Denmark, 'b Solar Energy Programme, Risø National Laboratory for Sustainable Energy, Technical University of Denmark, PO 49, DK-4000 Roskilde, Denmark, 'Department of Basic Sciences and Environment, Faculty of Life Sciences, University of Copenhagen, 1871 Frederiksberg C, Denmark, and ${ }^{\mathbf{d}}$ Teijin Aramid BV, Fibre Physics Group, PO 5153, 6802 ED Arnhem, The Netherlands. Correspondence e-mail: brian@stack.nl
\end{abstract}

\section{Introduction}

The effects of stress and strain on the internal structure of polymer fibres, such as poly( $p$-phenylene terephthalamide) (PPTA, 'aramid') with trade names Twaron and Kevlar, ${ }^{1}$ have been a topic of intense scrutiny since the inception of such fibres. Given the remarkable tensile properties of these fibres, it is of significant interest to understand the structure-property relationships that give the fibres these characteristics. Elucidation of the structural behaviour during and after application of tensile stress forms a key part of this endeavour. Simultaneous heating can significantly affect the tensile properties, and this combination of heating during the application of stress is used to produce a variety of fibres with a range of different tensile properties. Any structural insight into the nanostructural behaviour of the fibres during this process may aid understanding of their structure-property relationships considerably.

Small-angle X-ray scattering (SAXS) can be used to measure morphological aspects of the nanostructure (in particular the nanovoids) inside the fibres. Among the parameters that can be extracted are the size distribution parameters of the length and width of the voids and the orientation distribution parameters (Pauw et al., 2010). With this methodology in place, it has become possible to study in detail the behaviour of the nanostructure during the application of stress and heating.

Such investigations require an instrument that is (ideally) designed to be transported and installed at a SAXS beamline with a minimum of effort (i.e. having the aspect of portability), and is capable of applying heat and a large amount of stress to a fibre. Fibre tensile equipment also requires specialized clamping mechanisms to prevent

\footnotetext{
$\overline{1}$ Twaron and Kevlar are trademarks of Teijin Aramid BV and DuPont, respectively.
}

unwanted slippage or premature failure of the fibre at the clamping point (BISFA, 2002). In addition to this, the maximum elongation at break for the fibres studied here is at most a few percent. The high stresses and low strains, combined with the requirement for a small footprint (portability), make the default method of stress control through application of strain a difficult feat.

With this in mind, a novel pneumatic tensile stage is presented here, which meets the required characteristics of being compact, in vacuum, stress imposing and capable of radiative heating. The envisioned (and demonstrated) usage is in SAXS equipment, but the stage can be adapted to suit other analysis tools. The application to an aramid fibre is demonstrated.

\section{Stage design}

The design of fibre clamps for aramid fibres was optimized. A stainless steel bollard with a shallow guiding groove is used to partially relieve the load of the clamp on the fibres, by guiding them around a $180^{\circ}$ turn with a turn radius of $20 \mathrm{~mm}$. The fibres are subsequently clamped to the bollard through the use of two separate aluminium shoes. Care is taken that no burrs or sharp edges exist on either the bollard or the shoe, as this would lead to premature fibre failure. A schematic of one of the fibre clamps is shown in Fig. 1.

One clamp position remains fixed within the enclosure, while the other clamp is pulled by two pneumatic pistons. By controlling the amount of pressure on the pistons, the stress on the fibre can be directly controlled (up to a maximum of about $500 \mathrm{~N}$ can be applied). At this point, however, no strain feedback mechanism is in place. The pressure on both sides of the pistons is controlled using pressure reducers and digital manometers.

At the measurement position within the enclosure, a $20 \times 20 \times$ $18 \mathrm{~mm}$ aluminium heater surrounds the fibre, allowing for radiative 
heating of the fibre within the enclosure. The heater rests on a Teflon insulating base and is heated with a single heating cartridge coupled to a temperature controller. The X-ray beam path runs through a hole in the heater.

The entire enclosure is evacuated, so that the fibre is kept devoid of all moisture. This is optimal when studying aramid fibres, as moisture in the fibres complicates the scattering pattern analysis (Saijo et al., 1994). The X-ray transparent windows are constructed from $10 \mu \mathrm{m}$ thick circular sheets of mica, obtained from JJ-XRay (Denmark), which are sandwiched between two aluminium plates with o-rings. The open enclosure is shown in Fig. 2.

\section{Proof-of-principle experiments}

\subsection{Sample preparation}

The commercially available aramid fibre Twaron 1000 was employed for proof-of-concept tests. This fibre consists of a bundle of 1000 PPTA filaments with a diameter of $12 \mu \mathrm{m}$, and has been obtained from Teijin Aramid BV. The sample does not contain spin finish, a coating usually applied on the yarn surface to improve various yarn properties.

All fibre samples were prepared a minimum of one week before the synchrotron SAXS measurements and were dried in vacuum before transportation. The samples were transported in a desiccator kept dry with silica gel. Upon arrival at the synchrotron facility, the samples were again kept in an evacuated enclosure to prevent the uptake of moisture. Exposure of the fibre to air during mounting was

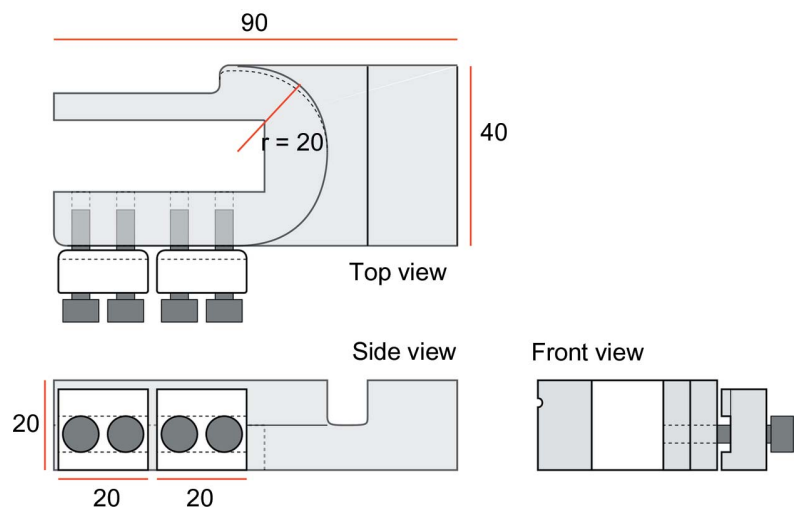

Figure 1

Top, side and front views of the mobile clamp (to which the pistons are attached). The shallow groove (indicated with the dotted line) extends halfway through the bend. The slot in the centre is used to guide the clamp motion.

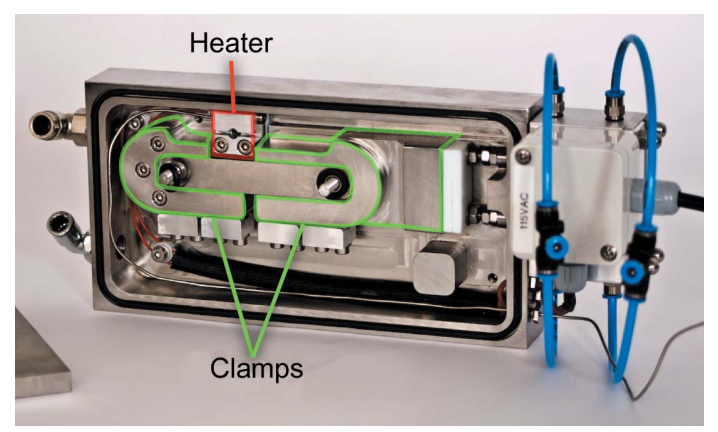

Figure 2

Internals of the tensile stage. Vacuum connections are shown on the left side, pneumatic pistons and electrical connections are on the right side. X-rays pass through the centre hole of the aluminium heating block. kept to a minimum and after mounting the fibres were again kept in an evacuated state for $10 \mathrm{~min}$ before commencement of the measurement series.

\subsection{Beamline details}

Synchrotron experiments were performed at the BW4 beamline at the DORIS synchrotron in Hamburg, Germany. The collimation was a square collimation, $0.4 \times 0.4 \mathrm{~mm}$ in size. The wavelength used was $1.381 \AA$, with a sample-to-detector distance of $1.560 \mathrm{~m}$. The scattering patterns were recorded using a Marresearch 165 CCD detector, quantized into $2048^{2}$ pixels, each $79.1 \times 79.1 \mu \mathrm{m}$ in size. Transmission values were determined using a beamstop-mounted direct beam intensity monitor. The data were corrected for background scattering effects.

3.2.1. Data analysis. The data were analysed using a full twodimensional scattering pattern analysis model, described elsewhere (Pauw et al., 2010).

Before analysis, the data were binned with a binning size of $4 \times$ 4 pixels. The model intensity values were computed on a polar grid spanning $90^{\circ}$ in $\psi(\psi$ is the detector azimuthal angle; symmetry is used to extend the range from $90^{\circ}$ in $\psi$ to $180^{\circ}$ in $\psi$ ). The fit was performed over $180^{\circ}$ in detector angle $\psi$ and one decade in $q$ [ $q=$ $(4 \pi / \lambda) \sin \theta$, where $\theta$ is half the scattering angle and $\lambda$ is the wavelength of the incident radiation], from $0.023 \leq q \leq 0.23$ using 1350 points. A least-squares fit is used to determine the model parameters, allowing all parameters to be evaluated simultaneously. Since the statistical errors of the collected intensity values are not known, an unweighted least-squares fit is used.

\section{Stage performance}

Using the tensile stage, four treatments were investigated. Firstly, the effects of the application of stress at room temperature on the nanostructure were investigated, secondly the effects of heating without application of stress, thirdly the application of stress on a hot fibre and lastly the effects of an increase in temperature on a stressed fibre.

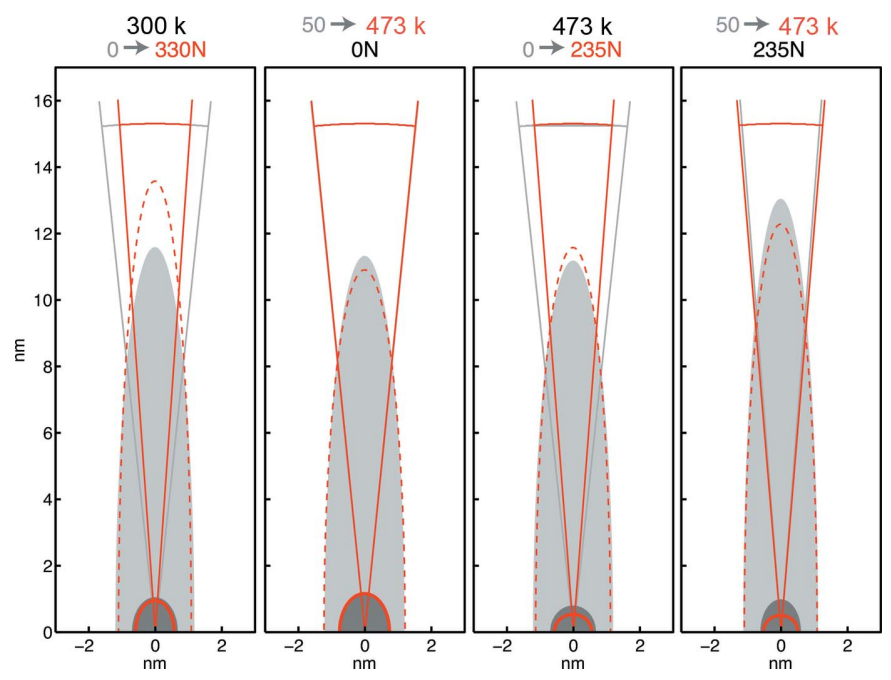

Figure 3

Schematic representation of void distribution parameters before and after application of four different treatments of Twaron 1000 fibre. The grey colour indicates the initial state and red indicates the final stage. Ellipsoids indicate the mode (small) and $90 \%$ confidence interval (large) of the void size distributions. The angular section emanating from the centre of the ellipsoids indicates the FWHM of the void orientation distribution. The fibre axis is vertical. 
Diagrams are given in Fig. 3 showing the effects of each treatment on the void structure in the fibres. These diagrams show the initial state in grey and the final state in red. The dark-grey filled ellipsoids show the mode of the size distribution in the initial state. The solid red outlined (but unfilled) ellipsoids show the mode of the distribution in the final state. The light-grey and dashed ellipsoids indicate the upper limit of the $90 \%$ confidence interval of the size distributions. The displayed angle emanating from the centre of the ellipsoids indicates the full width at half-maximum of the rotational smearing, which is related to the degree of misalignment of the voids with respect to the fibre axis.

Through application of stress on the fibre kept at room temperature, a clear increase in void length can be observed in correspondence with a slight decrease in void width. The orientation distribution width simultaneously shows a decrease, indicating an improved void alignment within the stressed fibre.

The effects of the application of increased temperature (to $473 \mathrm{~K}$ ) on the unstressed fibres show a slight decrease in void length, and hint towards a minor increase in void width. The orientation distribution remains unaffected.

The third treatment, where the stress is increased after heating the fibre to $473 \mathrm{~K}$, shows a combination of the aforementioned effects; an increase in orientation due to the applied stress, a decrease of the mode of the distribution (but an increase in the $90 \%$ confidence interval indicating increased polydispersity) and a decrease in void width. Thus it can be posited that the applied stress influences the orientation distribution, but that the elongation and narrowing effects of the stress are partially offset by the elevated temperatures.

Lastly, the increase of temperature on a stressed fibre (loaded to $\sim 235 \mathrm{~N}$ ) shows a very different effect, where the lengths are now significantly reduced when compared with the initial (stressed) state. A small reduction in width is also visible.

While no firm conclusions should be made on the basis of these preliminary data, the results do indicate that a wealth of heretofore difficult to obtain information can be accessed using the in situ tensile stage. The experiments have elucidated where the stage can be improved in order to widen the operating ranges and increase overall performance of the stage.

The performance of the tensile stage has met expectations. Sufficient tensile stress can be applied to the fibres, reaching and exceeding the force required for failure. Failure of the fibre occurs at various places on the fibre and does not appear to be localized to the clamps. This indicates that the grooved clamp design with a bollard radius of curvature of $20 \mathrm{~mm}$ is sufficient for attaining a good loading of stress onto the aramid fibres (although for less flexible fibres, larger radii of curvature may be needed). Control of the applied pressure through manometers on both piston sides proved to be an effective control method.

The heater reached design temperatures but is unable to reach temperatures beyond $523 \mathrm{~K}$. It is therefore unable to reach temperatures at which the aramid material may show more pronounced morphological effects. This is partially due to the heat losses through the two stainless steel mounting bolts, but also due to the choice of heater material (as aluminium becomes very ductile at higher temperatures) and base material (at high temperatures, Teflon will start to decompose). A reconsideration of the used materials [i.e. switching to a copper heater and ceramic isolating material, a design similar to that described by Kehres et al. (2010)] will be required for reaching higher temperatures. Additionally, the speed of the radiative heat transfer using this design will have to be tested. This can be measured by the correlation of the crystal lattice changes upon heating.

Another, more drastic, improvement would be the replacement of the pneumatic pistons with hydraulic ones. Doing so will increase the dynamic range of the tensile stage considerably, as this is now limited by the minimum threshold pressure required for the pneumatic pistons. At the cost of added complexity, a strain gauge and pressure transducer may aid in correlating the measurements to accurate stress and strain measurements. Lastly, if a vacuum sample chamber is available, the setup dimensions can be further reduced in size, as then the vacuum enclosure of this stage will no longer be required.

\section{Conclusions}

An interesting field of research becomes easily accessible with this stage, where changes in internal structure can be observed upon application of stress while applying heat. The example application shows that this induces significant changes in the internal structure of PPTA fibres. The effects of simultaneous heating and stretching appear to be a combination of the effects of application of either heating or stretching, but their counteracting effects mean that in situ experiments such as these are necessary to obtain insight into the actual structure while both are applied.

The stage offers a compact in situ high-stress low-strain fibre tensile stage with heating capability. Several minor and major improvements to the design have been suggested, the most effective design change being a change in heater material.

The authors would like to thank HASYLAB and the BW4 staff for the measurement time and the assistance, and Danscatt for travelling funds. Kirsi Svedström, Paavo Penttilä, Thomas Hecksher and Fengxiao Guo have also been instrumental in the success of the measurements with their hard work. Furthermore, gratitude is extended towards Teijin Aramid BV for their knowledge, funding and samples. K. D. Joensen of JJ X-ray Systems A/S has been very helpful in assisting with insight, design tips and sheets of mica. Additional thanks are extended to Michael Lindaa at the machine shop at the Technical University of Denmark for CNC milling and assembly of the tensile stage.

\section{References}

BISFA (2002). Testing Methods for Para-aramid Filament Yarns. BISFA, Brussels, Belgium.

Kehres, J., Andreasen, J. W., Krebs, F. C., Molenbroek, A. M., Chorkendorff, I. \& Vegge, T. (2010). J. Appl. Cryst. 43, 1400-1408.

Pauw, B. R., Vigild, M. E., Mortensen, K., Andreasen, J. W. \& Klop, E. A. (2010). J. Appl. Cryst. 43, 837-849.

Saijo, K., Arimoto, O., Hashimoto, T., Fukuda, M. \& Kawai, H. (1994). Polymer, 35, 496-503. 